\title{
The differential impact of oral poliovirus vaccine formulation choices on serotype-specific population immunity to poliovirus transmission
}

\author{
Kimberly M. Thompson ${ }^{*}$ and Radboud J. Duintjer Tebbens
}

\begin{abstract}
Background: Prior analyses demonstrated the need for some countries and the Global Polio Eradication Initiative (GPEl) to conduct additional supplemental immunization activities (SIAs) with trivalent oral poliovirus vaccine (tOPV) prior to globally-coordinated cessation of all serotype 2-containing OPV (OPV2 cessation) to prevent the creation of serotype 2 circulating vaccine-derived poliovirus (CVDPV2) outbreaks after OPV2 cessation. The GPEl continues to focus on achieving and ensuring interruption of wild poliovirus serotype 1 (WPV1) and making vaccine choices that prioritize bivalent OPV (bOPV) for SIAs, nominally to increase population immunity to serotype 1, despite an aggressive timeline for OPV2 cessation.
\end{abstract}

Methods: We use an existing dynamic poliovirus transmission model of northwest Nigeria and an integrated global model for long-term poliovirus risk management to explore the impact of tOPV vs. bOPV vaccine choices on population immunity and cVDPV2 risks.

Results: Using tOPV instead of bOPV for SIAs leads to a minimal decrease in population immunity to transmission of serotypes 1 and 3 polioviruses, but a significantly higher population immunity to transmission of serotype 2 polioviruses. Failure to use tOPV in enough SIAs results in CVDPV2 emergence after OPV2 cessation in both the northwest Nigeria model and the global model. Despite perceptions to the contrary, prioritizing the use of bOPV over tOPV prior to OPV2 cessation does not significantly improve serotype 1 population immunity to transmission.

Conclusions: Immunization leaders need to focus on all three poliovirus serotypes to appropriately manage the risks of OPV cessation in the polio endgame. Focusing on population immunity to transmission to interrupt WPV1 transmission and manage pre-OPV cessation risks of CVDPVs, all countries performing poliovirus SIAs should use tOPV up until the time of OPV2 cessation, after which time they should continue to use the OPV vaccine formulation with all remaining serotypes until coordinated global cessation of those serotypes.

Keywords: Polio, Eradication, Risk management, OPV, Vaccine choice

\section{Background}

National immunization programs and the Global Polio Eradication Initiative (GPEI) continue to manage risks associated with the polio endgame, including the risks of using oral poliovirus vaccine (OPV) to end all transmission of wild polioviruses (WPVs). The GPEI currently plans to globally-coordinate cessation of all serotype-2 containing OPV (i.e., OPV2 cessation) according to an aggressive timeline with a target date of April 2016 [1]. Prior modeling studies demonstrated the need for

* Correspondence: kimt@kidrisk.org

Kid Risk, Inc., 10524 Moss Park Rd., Ste. 204-364, Orlando, FL 32832, USA sufficient supplemental immunization activities (SIAs) with OPV prior to globally-coordinated OPV cessation to prevent the creation of circulating vaccine-derived poliovirus (cVDPV) outbreaks after OPV cessation [26]. The planned addition of a single dose of inactivated poliovirus vaccine (IPV) given at the time of the third trivalent (tOPV) dose of routine immunization (RI) for countries currently using OPV-only for immunization [1] appears to offer only marginal benefits for areas most at risk of cVDPVs after OPV cessation [3]. While clinical trials suggest that IPV boosts intestinal immunity more than OPV in individuals with prior immunity induced 
by a live poliovirus (LPV, i.e., WPV, OPV, cVDPV, or any OPV-related viruses) $[7,8]$, models consistent with this observation at the individual level show a minimal impact of IPV on immunity to poliovirus transmission at the population level $[3,5,6]$. This occurs because while IPV protects individual vaccine recipients from developing paralysis if they become infected with an LPV, IPV use leads to relatively little impact on fecal-oral poliovirus transmission in previously susceptible individuals and therefore in populations with conditions conducive to fecal-oral poliovirus transmission. Conditions associated with relatively higher-income countries (i.e., low population density, good hygiene, relatively greater role of oropharyngeal transmission) [9] increase the relative impact of IPV on population immunity to poliovirus transmission, but the minimal conditions for IPV to provide sufficient population immunity to prevent poliovirus transmission remain uncertain. The recent experience with WPV1 transmission in Israel despite high RI coverage with IPV provided valuable context [10].

Following a shift in GPEI policy that began in the mid2000s from exclusive use of tOPV to the use of monovalent OPV (mOPV) in SIAs in endemic countries with the expectation that using mOPV serotype 1 (mOPV1) would soon stop wild poliovirus (WPV) serotype 1 (WPV1) transmission in endemic areas [11], OPV formulation vaccine choices for SIAs became an important factor in the overall population immunity to transmission for each of the three poliovirus serotypes. The GPEI and some statistical analyses $[12,13]$ define population immunity as vaccine-induced immunity to disease among children under 5 years of age with non-polio acute flaccid paralysis. This characterization of population immunity to disease does not account for immunity derived from exposure to LPVs in the environment and it ignores the contributions to transmission of individuals of all ages immune to disease who can still participate in asymptomatic transmission [14]. The resulting vaccine choices for SIAs focus on the premise that competition between serotypes in tOPV in individuals leads to "vaccine failure" for serotypes 1 and 3 due to relatively lower take rates for individuals receiving tOPV than for those receiving mOPV $[11,13,15]$. In contrast with this individual vaccineinduced characterization of population immunity, our characterization of population immunity to poliovirus transmission uses a dynamic disease model to characterize immunity to poliovirus transmission of all three serotypes for all individuals in the entire population based on their exposure history to vaccines and circulating LPVs and focuses on the level of population immunity needed to stop viral transmission $[9,14]$.

Despite high expectations, the single serotype (mOPV1) strategy failed to achieve WPV1 interruption and led to outbreaks with serotype 3 WPV (WPV3), which then motivated the introduction of serotype 3 monovalent OPV (mOPV3) followed by bivalent OPV (bOPV, serotypes 1 and 3) for SIAs. Recent modeling suggested that the strategy pursued (i.e., the introduction of mOPVs then bOPV) delayed the interruption of WPVs in India [5]. Continued failures to achieve and maintain high population immunity to transmission delay eradication and allow WPV importations and cVDPV emergences and importations to cause outbreaks [16]. Despite the somewhat lower relative take rates for serotypes 1 and 3 characterized as "vaccine failure" for tOPV, the experience and models of the last endemic areas in which clusters of undervaccinated children repeatedly miss immunizations and sustain WPV transmission suggests that "failure to vaccinate" represents the fundamental problem $[4-6,17]$, confirmed for Nigeria recently by a subsequent independent analysis [18]. For example, despite the large numbers of SIAs conducted each year in northwest Nigeria, between 2010 and 2014, 47 \% (121/256) of all confirmed WPV1, WPV3, and cVDPV2 cases reported receipt of 2 OPV doses or fewer, which may include some heterotypic OPV doses (e.g., bOPV doses received by patients paralyzed by cVDPV2).

Modeling particularly indicates the need for more tOPV SIAs to prevent serotype 2 cVDPV (cVDPV2) outbreaks after OPV2 cessation [4]. The GPEI continues to focus on achieving and ensuring interruption of WPV1 transmission and making vaccine choices that prioritize the use of bOPV for SIAs, nominally to increase population immunity to disease for serotype 1 , despite an aggressive timeline for OPV2 cessation. However, achieving and maintaining polio eradication requires permanently stopping and preventing transmission, which requires a focus on population immunity to transmission, not a focus on population immunity to disease. This paper aims to explore the trade-offs in population immunity to transmission for the three poliovirus serotypes for tOPV or bOPV use in SIAs.

\section{Methods}

We use an existing differential equation-based dynamic poliovirus transmission and OPV evolution model [9] (i.e., the DEB model) to characterize the impacts of different vaccine choices for SIAs in northwest Nigeria [4, $6,17,19]$ and an integrated global model of long-term poliovirus risk management (i.e., the global model) [20] to explore the impacts of tOPV vs. bOPV vaccine choices on global population immunity to poliovirus transmission and cVDPV risks. Briefly, the DEB model dynamically tracks individuals as they move between immunity states because they acquire immunity from maternal antibodies, successful IPV vaccination, successful OPV vaccination or infection due to contact with an OPV vaccine recipient, or other LPV exposure, and lose immunity in the absence of further vaccinations or 
infections due to waning. We define successful vaccination as receipt of a vaccine that "takes" (i.e., typically approximated by seroconversion as measured in clinical trials), with take rates that depend on the vaccine and setting. We determined situation-specific average perdose take rates for all available poliovirus vaccines by model calibration within ranges from the literature [9, $21]$. In some cases, this included adjustments to account for study limitations (e.g., different settings and vaccines used in clinical trials than in the modeled population, possible interference with maternal antibodies or secondary OPV infections with study results). In the DEB model, cVDPVs emerge when population immunity to transmission becomes so low that OPV-related viruses introduced through RI or SIAs can sustain transmission in the population and evolve to successive reversion stages with increasingly high basic reproduction numbers ( $R_{0}$ values) and paralysis-to-infection ratios (PIRs). When the prevalence in the last of 20 reversion stages chosen to adequately represent the OPV evolution process $[9,22]$ exceeds a given transmission threshold, then fully-reverted VDPVs with assumed equal $R_{0}$ and PIR as homotypic WPV circulate in the population and a cVDPV outbreak can occur.

We characterize population immunity to poliovirus transmission in two different but related ways $[4,6]$. The mixing-adjusted effective immunity proportion (EIPM) represents the proportion of immune individuals in a population, weighted by the relative potential contribution to transmission for their immunity state and the extent to which they mix with other age groups or connected subpopulations. If EIPM remains above its threshold EIP* $=1 /\left(1-\mathrm{R}_{0}\right)$, then transmission eventually stops, while for EIPM $<$ EIP* transmission can continue and imported viruses can establish transmission. However, because EIP* depends on $R_{0}$, which changes over time and varies by serotype and setting, for this analysis we focus on the mixing-adjusted net reproduction number $\left(R_{n}\right)$, which equals $R_{0} \times(1-E I P)$ and represents the average number of secondary infections generated by a single infectious individual, taking into account mixing between age groups and subpopulations and the relative potential contribution to transmission of all individuals in the population. The threshold $\left(\mathrm{R}_{\mathrm{n}}{ }^{*}\right)$ equals 1 for any $\mathrm{R}_{0}$, serotype, setting, or point in time $[4,19]$, such that if $R_{n}>1$, then each new infection generates at least one new infection and transmission of existing or imported poliovirus can continue, but if $R_{n}<1$ for a long enough period of time then transmission eventually dies out.

To explore SIA vaccine choices in the northwest Nigeria DEB model, we adopt all inputs from the most recent model update $[4,19]$. This includes assumed continuation of the status quo of 9 annual SIAs until globally-coordinated cessation of serotype 1 and serotype 3-containing OPV (OPV13 cessation) on April 1,2019 . The model further assumes OPV2 cessation on April 1, 2016, which switches RI and SIAs from tOPV to bOPV. Given very low RI coverage (i.e., of $26.4 \%$, $22.2 \%, 18.1 \%$, and $13.9 \%$ with dose 0 (i.e., birth), 1, 2, and 3, respectively as of 2013) [23], uncertainty about timing, and scale of IPV use going forward, we previously demonstrated very limited impact of IPV on population immunity and cVDPV risks in settings like northwest Nigeria $[3,5,6]$. Consequently, we do not include IPV use in the northwest Nigeria DEB model, although Nigeria began introducing it in SIAs in some limited areas. We report $R_{n}$ as a function of time for different options that use tOPV for between 0 and 9 of the annual SIAs between January 1, 2015 and OPV2 cessation. To spread out the impact of tOPV SIAs over the year while concentrating as many tOPV SIAs as possible in the months before OPV2 cessation on April 1, 2016, we change successive SIAs from bOPV to tOPV in the following order: March, November, August, January, May, December, June, September, April. For example, the option of 3 annual tOPV SIAs implies tOPV use during the March, November, and August SIAs.

The global model divides the world into 710 subpopulations of approximately 10 million people in 2013 and uses the DEB model to track infections and population immunity to transmission in each subpopulation [20]. The global model groups each subpopulation into 9 global regions consisting of variable numbers of epidemiological blocks that in turn consist of 10 subpopulations of equal size to simulate random exportations from subpopulations to other subpopulations in the same block (i.e., representing $96 \%$ of all exportations) or other blocks (i.e., representing $4 \%$ of all exportations, including $3.5 \%$ within the same region and $0.5 \%$ elsewhere). As in the DEB model, cVDPVs can emerge endogenously if population immunity to transmission becomes low enough as long as OPV virus from immunization (including RI) or importations transmit in any subpopulation. The global model characterizes other long-term risks after OPV cessation stochastically, but given our focus on short term population immunity to transmission we ignore those risks in this analysis. The subpopulations in the global model reflect conditions related to poliovirus transmission and vaccination similar to real conditions that exist throughout the world and consistent with available global data [24], but at a more abstract level amenable to our global mixing characterization and simplification of the complex RI and SIA histories in each country $[9,20,21]$. The global model includes 4 blocks with conditions like the last 4 global reservoirs of indigenous WPV1 and WPV3 transmission, which each include an under-vaccinated subpopulation. These 4 subpopulations sustain WPV1 and WPV3 the longest in 
the model, and because of their very low RI coverage and poor SIA quality they also represent the highest risk areas of cVDPV emergence after OPV cessation.

With respect to SIAs, the global model assumes that blocks increase the annual SIA frequency by one per year each year until they eliminate all indigenous WPV transmission. After elimination of all indigenous WPVs from a block and in the absence of any detected outbreaks, subpopulations conduct between 0 and 6 annual preventive SIAs, depending on their RI coverage with 3 or more non-birth doses (POL3) and $R_{0}$ [20]. For reference, Table 1 provides the specific SIA schedule assumptions. The global model assumes that between 2010 and January 1, 2015, 2-5 annual SIAs in populations that conduct more than 1 annual SIA use bOPV and the remainder use tOPV (Table 1). We assume tOPV intensification starts on January 1, 2015 and switches between 1 and 2 annual SIAs from bOPV to tOPV until OPV2 cessation. We consider the implications of tOPV intensification on all three serotypes by reporting the $R_{n}$ of all three serotypes at the time of OPV2 cessation with or without tOPV intensification.

We further explore the option of simultaneously coordinating cessation of all 3 OPV serotypes (OPV123 cessation) on April 1, 2019. Specifically, we compare the
$R_{n}$ of each of three serotypes on April 1, 2019 for an option of continued tOPV intensification (i.e., continued use of both tOPV and bOPV for SIAs, as specified in Table 1) until OPV123 cessation with the corresponding $R_{n}$ for an option of exclusive tOPV use for RI and SIAs from January 1, 2017 until OPV123 cessation. We focus the comparison on 165 subpopulations affected by tOPV intensification, which includes all subpopulations with a POL3 of less than 0.9. For all options, the global model assumes that subpopulations that used OPV-only as of 2013 add a single IPV dose co-administered with the third non-birth OPV RI dose on January 1, 2015, consistent with the current plan [1].

Table 2 summarizes our assumed take rates in the DEB and global models along with data from the only published clinical trials that directly compared current bOPV and tOPV vaccines [25, 26]. The clinical trial conducted in three sites in Central and Southern India reports seroconversion rates for all three serotypes among newborn children vaccinated with tOPV or bOPV at birth and again at 30 days [25]. Table 2 reports average per-dose take rates for tOPV and bOPV, calculated from the cumulative seroconversion rates after 2 doses (CS2) as $1-(1-C S 2)^{1 / 2}$. The seroconversion rate observed for serotype 2 in the bOPV arm of the trial may reflect a

Table 1 Planned, preventive (pSIA) SIA schedules used in the global model before and after OPV2 cessation in OPV-using blocks after interruption of indigenous wild poliovirus transmission in each block (based on Duintjer Tebbens et al. (2015) [20])

\begin{tabular}{|c|c|c|}
\hline Time period & RI coverage (POL3) & SIA schedule showing: vaccine (day(s) of year) \\
\hline \multirow[t]{7}{*}{ Before tOPV intensification on January 1, 2015} & 0.05 or 0.1 & tOPV $(0,40) ;$ bOPV $(80,140,240,300)$ \\
\hline & 0.3 & tOPV $(0,40) ;$ bOPV $(80,140,240)$ \\
\hline & $0.6\left(R_{0} \leq 10\right)$ & tOPV (0); bOPV $(60,120)$ \\
\hline & $0.6\left(R_{0}>10\right)$ & tOPV $(0,40) ;$ bOPV $(80,140,240)$ \\
\hline & 0.9 & tOPV $(0)$ \\
\hline & $0.98\left(R_{0} \leq 10\right)$ & No SIAs \\
\hline & $0.98\left(R_{0}>10\right)$ & tOPV $(0)$ \\
\hline \multirow[t]{7}{*}{ During tOPV intensification (January 1, 2015 to April 1, 2016) } & 0.05 or 0.1 & tOPV $(0,40,80,300) ;$ bOPV $(140,240)$ \\
\hline & 0.3 & tOPV $(0,40,80) ;$ bOPV $(140,240)$ \\
\hline & $0.6\left(R_{0} \leq 10\right)$ & tOPV $(0,60) ;$ bOPV $(120)$ \\
\hline & $0.6\left(R_{0}>10\right)$ & tOPV $(0,40,80) ;$ bOPV $(140,240)$ \\
\hline & 0.9 & tOPV $(0)$ \\
\hline & $0.98\left(R_{0} \leq 10\right)$ & No SIAs \\
\hline & $0.98\left(R_{0}>10\right)$ & tOPV $(0)$ \\
\hline \multirow[t]{7}{*}{ After tOPV intensification (April 1, 2016 to OPV13 cessation) } & 0.05 or 0.1 & $\operatorname{bOPV}(0,40,80,140,240,300)$ \\
\hline & 0.3 & $\operatorname{bOPV}(0,40,80,140,240)$ \\
\hline & $0.6\left(R_{0} \leq 10\right)$ & $\operatorname{bOPV}(0,60,120)$ \\
\hline & $0.6\left(R_{0}>10\right)$ & $\operatorname{bOPV}(0,40,80,140,240)$ \\
\hline & 0.9 & bOPV (0) \\
\hline & $0.98\left(R_{0} \leq 10\right)$ & No SIAs \\
\hline & $0.98\left(R_{0}>10\right)$ & bOPV (0) \\
\hline
\end{tabular}


Table 2 Serotype-specific average per-dose take rates for tOPV and bOPV determined in the clinical trials that compared both vaccines, and assumed in the DEB and global models

\begin{tabular}{|c|c|c|c|c|c|c|}
\hline \multirow[t]{2}{*}{ Setting } & \multicolumn{3}{|c|}{ tOPV serotype } & \multicolumn{3}{|c|}{ bOPV serotype } \\
\hline & 1 & 2 & 3 & 1 & 2 & 3 \\
\hline \multicolumn{7}{|l|}{ Clinical trials } \\
\hline Central and Southern India [25], 2 doses at 0 and 30 days of age & 0.39 & 0.70 & 0.31 & 0.62 & 0.06 & 0.49 \\
\hline Bangladesh [26], 3-doses at 6, 10, and 14 weeks of age) & 0.57 & 0.65 & 0.51 & 0.70 & 0.06 & 0.65 \\
\hline \multicolumn{7}{|l|}{ Calibrated DEB model within ranges from literature $[17,21]$} \\
\hline Northwest Nigeria $[4,6,9,17,19]$ & 0.45 & 0.70 & 0.35 & 0.54 & 0 & 0.54 \\
\hline Northern India $[5,9,17,19]$ & 0.35 & 0.60 & 0.27 & 0.42 & 0 & 0.42 \\
\hline \multicolumn{7}{|l|}{ Global model assumptions based on calibrated DEB model [20] } \\
\hline Lowest tier (e.g., Northern India) & 0.35 & 0.60 & 0.27 & 0.42 & 0 & 0.42 \\
\hline Second tier (e.g., Northern Pakistan) & 0.40 & 0.65 & 0.32 & 0.50 & 0 & 0.50 \\
\hline Third tier (e.g., Northwest Nigeria) & 0.45 & 0.70 & 0.35 & 0.54 & 0 & 0.54 \\
\hline Fourth tier (e.g., Brazil) & 0.50 & 0.72 & 0.40 & 0.60 & 0 & 0.60 \\
\hline Fifth tier (e.g., Philippines, Turkey) & 0.55 & 0.73 & 0.45 & 0.70 & 0 & 0.70 \\
\hline Sixth tier (e.g., Russia, middle-income China) & 0.60 & 0.74 & 0.50 & 0.75 & 0 & 0.75 \\
\hline Seventh tier (e.g., upper-income China, Israel) & 0.65 & 0.75 & 0.55 & 0.80 & 0 & 0.80 \\
\hline
\end{tabular}

small heterologous serological response, or more likely represents secondary exposure to serotype 2 OPV virus given the intensity OPV exposure in India [27]. The study results translate into a relative reduction of approximately $35 \%$ in the average per-dose individual take rate for tOPV compared to bOPV after two doses (both administered at an age of some partially reduced susceptibility due to maternal antibodies) [28]. However, a recent study that compared bOPV, mOPV1, and tOPV in different schedules in Bangladesh suggests that the difference becomes smaller with subsequent doses, because serotype 2 -interference with serotype 1 and 3 seroconversion decreases as individuals become better protected to serotype 2 [26]. For a standard schedule of three doses at 6,10 , and 14 weeks of age, the reduction in the average per-dose individual seroconversion rates for serotypes 1 and 3 (calculated from the cumulative seroconversion rates after 3 doses (CS3) assuming 1-(1CS3 $)^{1 / 3}$ ) amounted to only approximately $20 \%$ for tOPV compared to bOPV. Figure 1 summarizes the results from a 1989 Brazil tOPV seroconversion study [29] that confirms that few children seroconvert to serotypes 1 and 3 after the first dose, while more than half seroconvert to serotype 2 (Fig. 1a). However, once the majority of children acquire immunity to serotype 2 after the first dose, serotype interference diminishes, resulting in increased serotype 1 and 3 seroconversion for subsequent doses. Consequently, the differences in cumulative seroconversion rates between serotype 3 and serotype 2 decrease with each successive dose, and those between serotype 1 and serotype 2 almost disappear altogether after 4 doses (Fig. 1b). While the numerical results will differ in places other than Brazil due to environmental and other factors [30], we should similarly expect increased serotype 1 and 3 seroconversion with successive tOPV doses as children develop serotype 2 immunity. This means that for populations, which include a mixture of individuals with different ages and immunization and exposure histories, assumptions about the overall take rate of the different serotypes must recognize that only a small fraction of tOPV SIA recipients did not previously seroconvert to serotype 2 . Thus, the receipt of bOPV as a first dose instead of tOPV as a first dose for bOPV SIAs only leads to serotype 1 or 3 seroconversion instead of serotype 2 seroconversion for a relatively small fraction of the population.

Taking into account the average effect on take of multiple tOPV doses, our model assumes closer average perdose take rates for serotypes 1 and 3 bOPV and tOPV than observed after 2 doses for very young children in the controlled trial in India (Table 2) [25], but similar relative average per-dose take rates as found in Bangladesh after 3 bOPV vs. tOPV doses [26]. For the northwest Nigeria DEB model, the estimates in Table 2 produce serotype-specific incidence results consistent with the evidence $[4,6,9,17,19]$. For the integrated global model, we extrapolated from the northwest Nigeria model and models from other situations, including northern India [5, $9,17,19]$, to assign take rate tiers for different blocks. To explore the impact of differences between tOPV and bOPV serotype 1 and 3 takes rates as large as reported after 2 doses in the clinical trial in India [25], we perform 

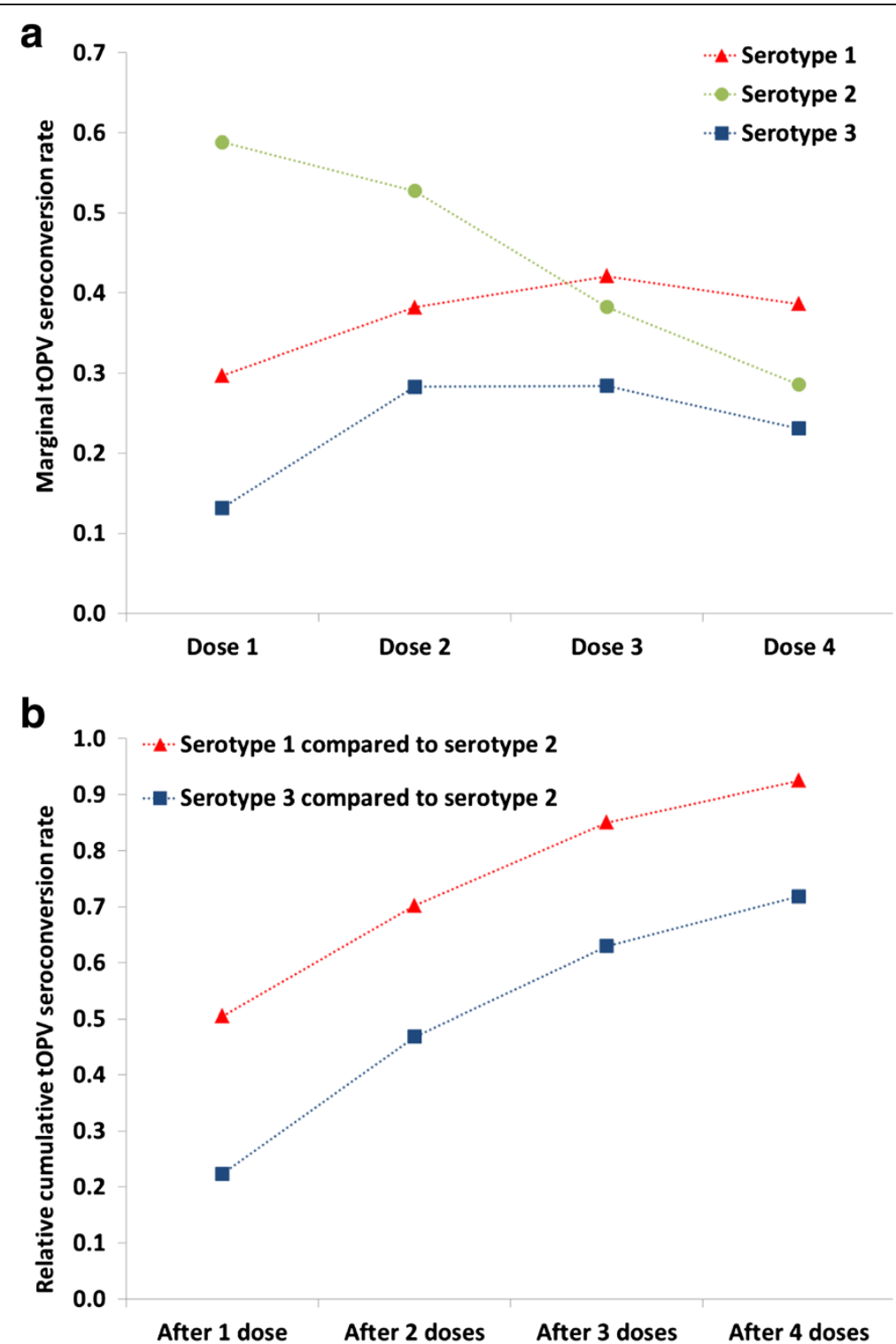

Fig. 1 tOPV response to the three serotypes, by number of doses, in a clinical trial in Brazil, 1989[30]. a Marginal seroconversion rates, defined as the incremental number of children seroconverting after each dose, divided by the number of children that did not yet seroconvert prior to the dose. $\mathbf{b}$ Relative cumulative tOPV seroconversion rates, defined as cumulative seroconversion rate for the given serotype after the given number of doses, divided by cumulative seroconversion rate for serotype 2 after the same number of doses

a sensitivity analysis that uses the observed average perdose rates as estimated from these data in Table 2 instead of our calibrated model estimates.

\section{Results}

Figure 2 shows population immunity to transmission of each serotype between 2015 and 2018 for different SIA vaccine choices in the northwest Nigeria model $[4,6,9$, $17,19]$. Higher $R_{n}$ values indicate more potential transmission per new infection and they correspond to lower population immunity (i.e., higher risk of transmission). Despite the assumed lower average per-dose serotype 1 and 3 take rates for tOPV compared to bOPV (Table 2), the high number of SIAs containing serotype 1 and 3 OPV results in very similar population immunity to transmission for serotype 1 and 3 regardless of the number of tOPV and bOPV SIAs (note the similarity of all curves Fig. 1a, c). Consequently, as long as northwest Nigeria maintains the same quality and frequency of SIAs [4], serotype 1 and 3 population immunity to transmission remain far enough below the threshold to minimize the risk of re-established WPV transmission from imported WPV or continued undetected WPV circulation [19], regardless of the proportion of the SIAs 
a Population immunity to serotype 1 poliovirus transmission

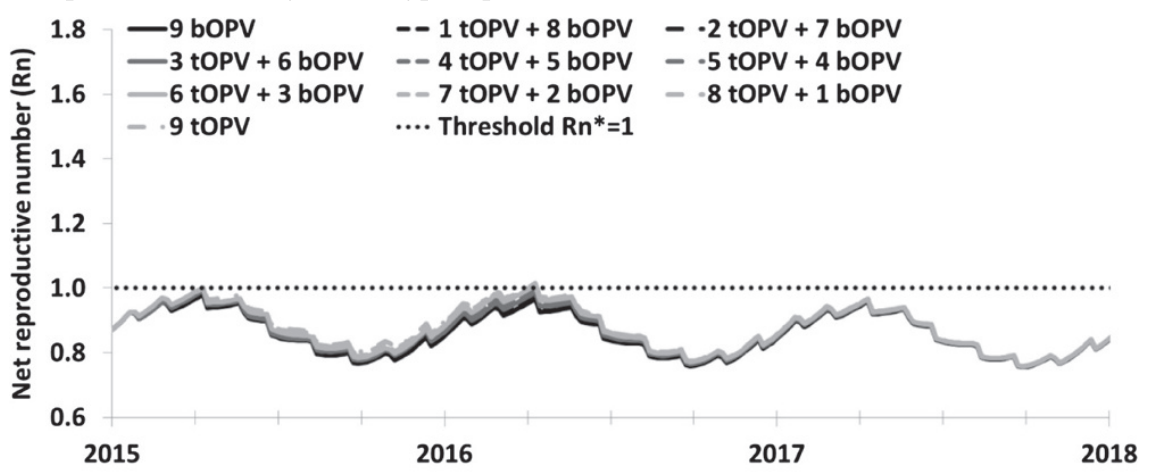

b Population immunity to serotype 2 poliovirus transmission

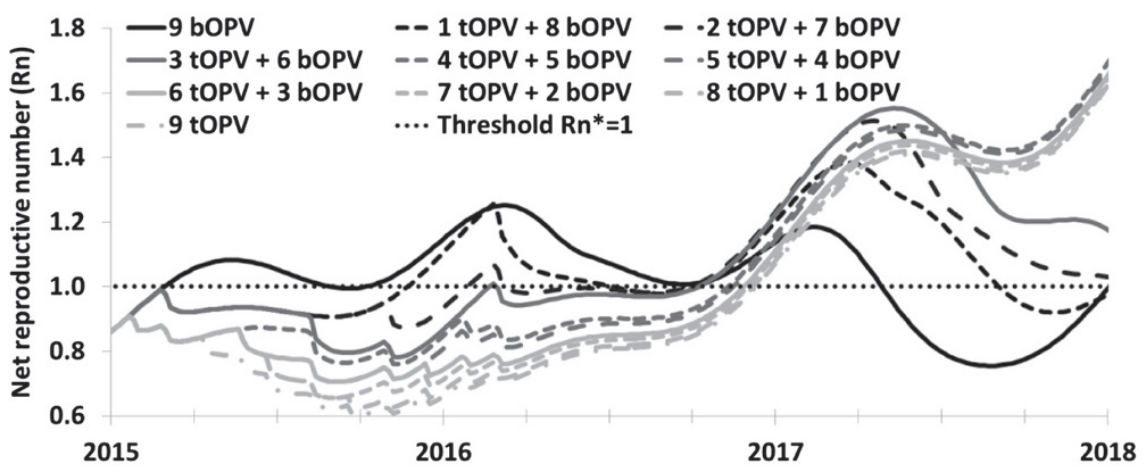

C Population immunity to serotype 3 poliovirus transmission

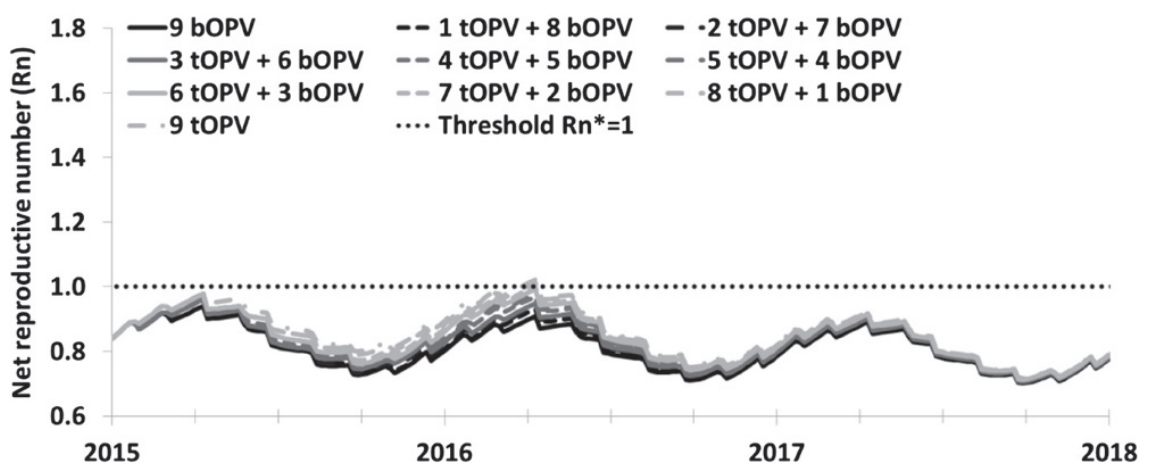

Fig. 2 Population immunity to transmission in northwest Nigeria for all 3 serotypes and different annual numbers of bOPV and tOPV SIAs. a Population immunity to serotype 1 poliovirus transmission. b Population immunity to serotype 2 poliovirus transmission. $\mathbf{c}$ Population immunity to serotype 3 poliovirus transmission

using tOPV or bOPV. In contrast, given that bOPV does not provide any immunity against serotype 2 , the number of tOPV SIAs greatly influences population immunity to serotype 2 poliovirus transmission (Fig. 1b). Specifically, for 3 or fewer annual tOPV SIAs between January 1, 2015 and the time of planned OPV2 cessation, population immunity to transmission at the time of OPV2 cessation does not become high enough to prevent cVDPV2 emergence after OPV2 cessation and/or to interrupt cVDPV2 transmission before OPV2 cessation. Due to the cVDPV2 outbreak that occurs with 3 or fewer annual tOPV SIAs, during 2017 the resulting viral circulation leads $R_{n}$ to decrease (i.e., population immunity to transmission increases due to cVDPV2 transmission) despite no OPV2 use, which represents a failure associated with OPV2 cessation that will require outbreak response. With 4 or more annual tOPV SIAs, $R_{n}$ continues to increase after OPV2 cessation because OPV2 use stops and all serotype 2 LPV transmission dies out during 2016. Prevention of cVDPV2s clearly represents the better option for achieving high population immunity to transmission 
for serotype 2 from a health perspective and in the context of managing global cVDPV2 risks, and this analysis demonstrates that tOPV vs. bOPV vaccine choices matter.

Figure 3 shows the population immunity results if the difference between average per-dose tOPV and bOPV take rates for serotypes 1 and 3 become as large as observed in the clinical trial in India after 2 doses (Table 2), thus assuming the entire population behaves like the limited population in the trial [25]. For serotype
2, the results do not change at all, because the serotype 2 tOPV take rate remains unchanged (Fig. 3b). For serotypes 1 and 3, the gap between the population immunity to transmission curves during 2015 and 2016 in Fig. 3a, c increases somewhat compared to those in Fig. 2a, c. However, the difference in population immunity to transmission remains significantly smaller than for serotype 2. Moreover, population immunity to transmission remains high enough to prevent reestablished WPV1 or WPV3 transmission during 2015

a Population immunity to serotype 1 poliovirus transmission

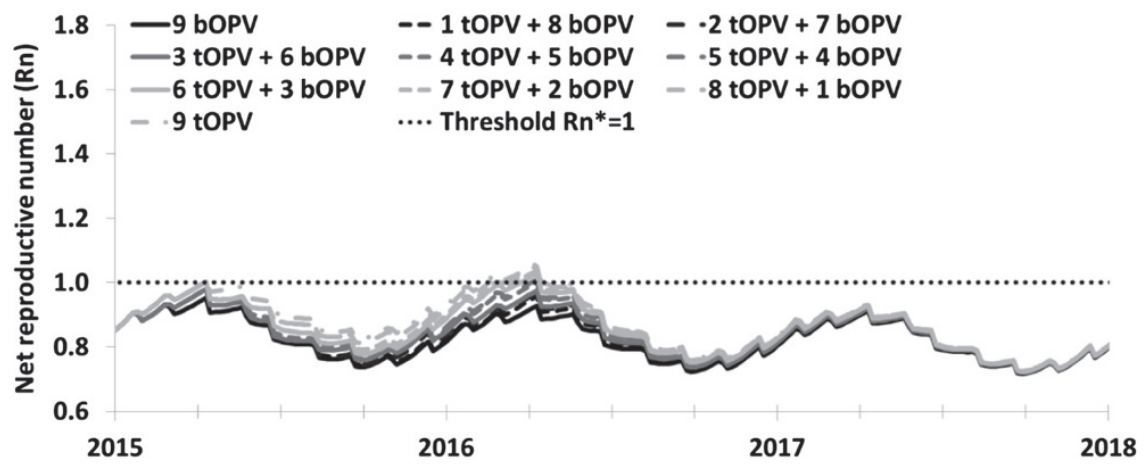

b Population immunity to serotype 2 poliovirus transmission

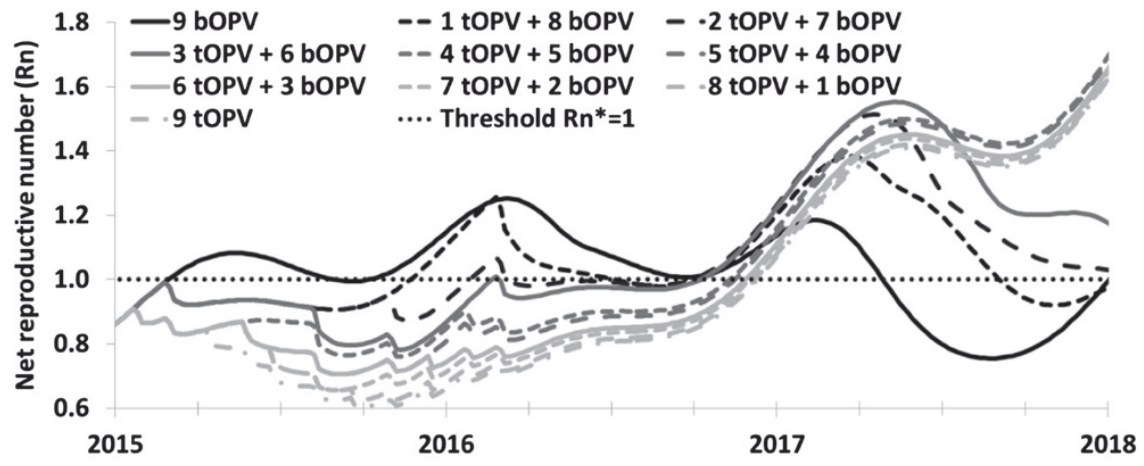

C Population immunity to serotype 3 poliovirus transmission

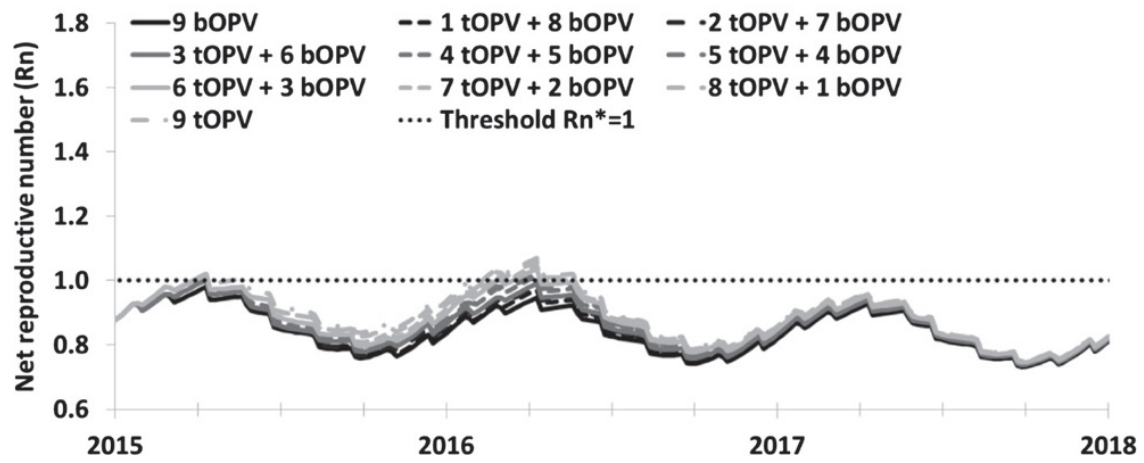

Fig. 3 Same model result as in Fig. 2, but with tOPV and bOPV take rates calculated directly from cumulative 2-dose seroconversion estimates reported by Sutter et al. (2010)[27] (see Table 1). a Population immunity to serotype 1 poliovirus transmission. b Population immunity to serotype 2 poliovirus transmission. c Population immunity to serotype 3 poliovirus transmission 
and 2016. After OPV2 cessation, the curves become similar again due to bOPV-only use for all options, which prevent cVDPVs of serotypes 1 and 3 after OPV13 cessation (not shown).

Figure 4 shows the results for different SIA vaccine choices in the global model [20]. The results confirm the observations from northwest Nigeria on a global scale. Comparison of options with more tOPV use to options with more bOPV use until OPV2 cessation (i.e., No tOPV intensification vs. tOPV intensification) shows minimal impacts on population immunity to transmission of OPV of serotypes 1 and 3 , with the $R_{n}$ values in all subpopulations remaining very close to the line indicating no difference between the two policies (Fig. 4a). However, for OPV serotype 2, Fig. 4a shows higher $R_{n}$ values in all subpopulations without tOPV intensification, implying a higher risk of cVDPV2 outbreaks after OPV2 cessation. Without tOPV intensification, although the $R_{n}$ values remain below 1 at OPV2 cessation, they increase after OPV2 cessation, allowing transmission of increasingly more transmissible OPV-related viruses. This leads to cVDPV2 outbreaks after OPV2 cessation in the global model without tOPV intensification that do not occur with tOPV intensification [20]. Fig. 4b shows that even exclusive tOPV use for all SIAs does not significantly reduce population immunity to transmission of OPV serotypes 1 and 3, while it results in a further marginal increase in population immunity to transmission of OPV serotype 2 compared to continued tOPV intensification. Figure $4 \mathrm{~b}$ assumes hypothetical simultaneous cessation of all 3 OPV serotypes (i.e., OPV123

a

Comparison of $\mathrm{R}_{\mathrm{n}} \mathrm{s}$ at time of OPV2 cessation on April 1, 2016

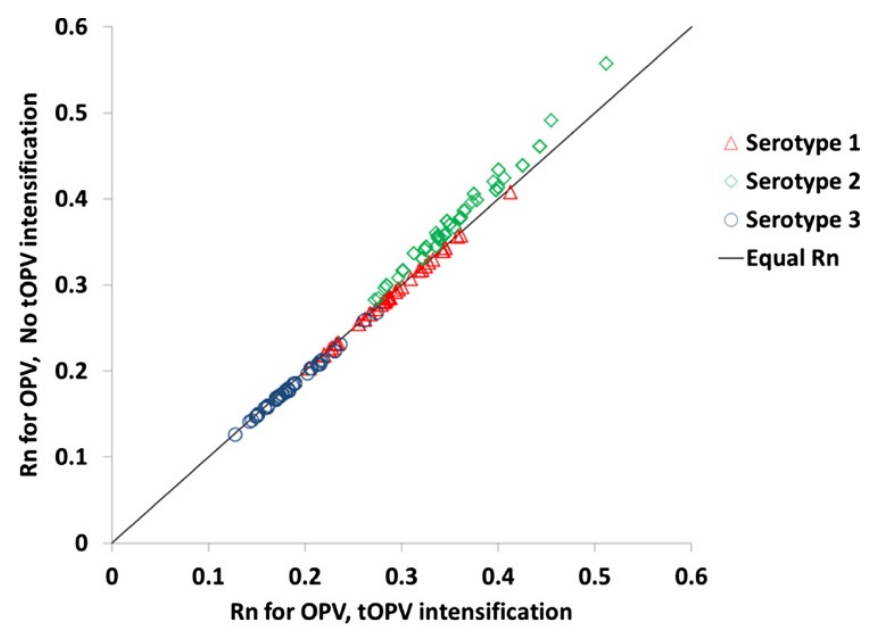

b Comparison of $\mathrm{R}_{\mathrm{n}} \mathrm{s}$ at time of hypothetical OPV123 cessation on April 1, 2019

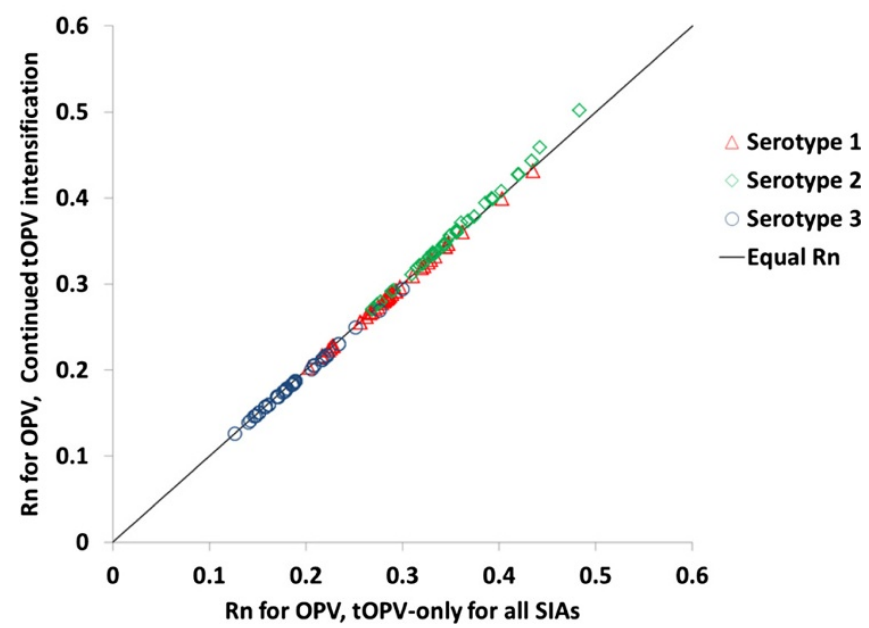

Fig. 4 Net reproduction number $\left(R_{n}\right)$ for OPV of each serotype for different SIA vaccine choices in 165 subpopulations affected by tOPV intensification in the global model [21] . a Comparison of $R_{n} s$ at time of OPV2 cessation on April 1, 2016. b Comparison of $R_{n} s$ at time of hypothetical OPV123 cessation on April 1, 2019 
cessation) in 2019 and does not lead to cVDPV outbreaks of any type after OPV123 cessation for either of the options. The absence of cVDPV1 and cVDPV3 outbreaks relates to the relatively lower $R_{0}$ values for OPV serotypes 1 and 3 and their slower evolution to fully-reverted VDPVs $[9,17,22]$, which results in faster die-out of serotypes 1 and 3 OPV-related viruses compared to serotype 2 OPVrelated viruses for any given population immunity level. However, as with OPV2 cessation, continued SIAs with OPV containing serotypes 1 and 3 up until OPV13 cessation remain necessary in populations with low RI coverage to prevent subsequent cVDPV1 and cVDPV3 outbreaks.

\section{Discussion}

Misplaced focus on vaccine failure and on vaccineinduced population immunity to disease $[11,13]$ continues to allow insufficient focus on the failure to vaccinate and the importance of managing population immunity to transmission of all three serotypes [2, 14, 16, 17]. Failing to achieve and maintain high population immunity to transmission leads to outbreaks and delays both WPV eradication and successful OPV cessation, which increases the overall costs of polio eradication. The complexity of simultaneously managing all 3 poliovirus serotypes requires sufficient use of poliovirus vaccine containing all 3 serotypes (i.e., tOPV or IPV). For countries with conditions conducive to intense transmission (e.g., high R0, relatively poor hygiene, and frequent fecal-oral contacts), even high coverage RI with IPV - only may prove insufficient to prevent transmission $[10,16]$. Although the relatively lower individual take rates for serotypes 1 and 3 for tOPV compared to bOPV may suggest a benefit associated with preferentially using bOPV for SIAs $[25,26]$, our results demonstrate the importance of the serotype 2 component in tOPV and the relatively small impact on population immunity to transmission of using bOPV for SIAs instead of tOPV. Moreover, with repeated tOPV immunizations, recipients develop serotype 2 immunity, which reduces serotype interference and makes subsequent tOPV doses de facto bOPV doses. Immunization leaders need to focus on all three poliovirus serotypes to appropriately manage the risks of both WPV eradication and OPV cessation in the polio endgame. Our analyses suggest that all countries performing polio SIAs should use tOPV up until the time of OPV2 cessation, after which time they should continue to use the OPV vaccine formulation with all remaining serotypes until coordinated global cessation of those serotypes as they manage population immunity to transmission.

While our model insights remain very robust to a range of assumptions about the differential impact of tOPV and bOPV on serotype 1 and 3 take rates, we note several limitations. First, the model does not explicitly characterize serotype interference but instead focuses on average per-dose take rates. However, using first-dose take rates instead would artificially decrease the impact of tOPV on serotype 1 and 3 poliovirus transmission, particularly in the context of frequent immunization contacts associated with either good RI coverage, or frequent SIAs, or both. The large difference associated with first-dose take rates would only apply in a situation in which children receive only one effective dose. Second, our model relies on sets of generic model inputs based on an expert literature review process $[22,28,31]$ that fits the evidence across a wide range of situations $[9,17]$ but that does not preclude the possibility that other combinations of generic model inputs may produce results overall consistent with the evidence. For example, the kinetics of waning remain uncertain and intestinal immunity may wane more steeply after 5 or more years [32] than assumed in our model [17] based on the limited evidence [28, 31, 33], which would result in more cVDPV emergences and cases both before and after OPV2 cessation. Thus, further empirical evidence and study of model uncertainties would further help inform decisions. Using different take rates (Fig. 3) than those based on the model calibration process led to some changes in the historical fit, and we did not explore the impact of take rates that vary by dose given that this would add significant complexity to the model (i.e., stratification of the model by dose histories in addition to immunity state, reversion stage, age group, serotype, and waning stage) without good data to support dosespecific take rate estimates in different settings. Nevertheless, the insights with respect to bOPV vs. tOPV in the context of repeated SIAs proved robust to the alternative assumptions about average per-dose take rates vs. the higher serotype interference associated with the first two doses, and we likewise expect robustness of the insights to different potential combinations of generic model inputs that remain consistent with the body of evidence on poliovirus immunity and transmission [28]. Third, the situation in northwest Nigeria or elsewhere may change in the future, particularly related to the confirmed cVDPV2 case reported in May 2015 [34], which should motivate Nigeria to use tOPV for more SIAs. If SIA frequency and/or quality decrease going forward, reemerging WPV transmission may occur, cVDPV2 transmission may not stop even with four annual tOPV SIAs, or cVDPVs could emerge after OPV cessation. Fourth, for the northwest Nigeria model, we did not model the evolving policies involving IPV SIAs, given their uncertain role in the immunization program. Studies on the impact of IPV SIAs on population immunity to poliovirus transmission of all three serotypes remains a topic of further research. Finally, all limitations associated with the models used in this analysis apply, and we refer to other publications for further discussion of those limitations $[9,20]$. 


\section{Conclusions}

Using tOPV instead of bOPV for SIAs leads to a minimal decrease in population immunity to transmission for serotypes 1 and 3, but a significantly higher population immunity to transmission for serotype 2 . Failure to use tOPV in enough SIAs results in cVDPV2 outbreaks after OPV2 cessation in both the northwest Nigeria model and the global model. Immunization leaders need to focus on all three poliovirus serotypes to appropriately manage the risks of OPV cessation in the polio endgame. Focusing on population immunity to transmission to manage pre-OPV cessation risks of cVDPVs, all countries performing poliovirus SIAs should use tOPV up until the time of OPV2 cessation, after which time they should continue to use the OPV vaccine formulation with all remaining serotypes until coordinated global cessation of those serotypes.

\begin{abstract}
Abbreviations
bOPV: Bivalent oral poliovirus vaccine(serotypes 1 and 3); $\operatorname{cVDPV}(1,2,3)$ : Circulating vaccine-derived poliovirus(serotype 1, 2, or 3, respectively); EIP*: Threshold effective immune proportion; EIPM: Mixingadjusted effective immune proportion; GPEl: Global Polio Eradication Initiative; IPV: Inactivated poliovirus vaccine; LPV: Live poliovirus; mOPV $(1,2,3)$ : Monovalent oral poliovirus vaccine (serotype 1, 2, or 3, respectively); OPV: Oral poliovirus vaccine; $R_{0}$ : Basic reproduction number; $\mathrm{R}_{\mathrm{n}}$ : Mixing-adjusted net reproduction number; RI: Routine immunization; SIA: Supplemental immunization activity; tOPV: Trivalent oral poliovirus vaccine; WPV(1,2,3): Wild poliovirus (serotype 1, 2, or 3, respectively).
\end{abstract}

\section{Competing interests}

The authors declare no competing interests.

\section{Authors' contributions}

KMT conceived of the study and participated in all aspects of the analysis and writing. RJDT led the modeling and participated in all aspects of the analysis and writing. Both authors read and approved the final manuscript.

\footnotetext{
Acknowledgements

We thank the Bill and Melinda Gates Foundation for providing a contract to Kid Risk, Inc. to support completion of this work under Work Order 4533-25298. The contents of this manuscript are solely the responsibility of the authors and do not necessarily represent the official views of the Bill and Melinda Gates Foundation.
}

Received: 21 May 2015 Accepted: 7 September 2015 Published online: 17 September 2015

\section{References}

1. World Health Organization. Global Polio Eradication Initiative: Polio Eradication and Endgame Strategic Plan (2013-2018). Geneva, 2013; Report No.: WHO/POLIO/13.02

2. Thompson KM, Duintjer Tebbens RJ. Modeling the dynamics of oral poliovirus vaccine cessation. J Infect Dis. 2014;210 Suppl 1:S475-84.

3. Duintjer Tebbens RJ, Thompson KM. Modeling the potential role of inactivated poliovirus vaccine to manage the risks of oral poliovirus vaccine cessation. J Infect Dis. 2014;210(S1):485-97.

4. Duintjer Tebbens RJ, Pallansch MA, Wassilak SGF, Cochi SL, Thompson KM. Combinations of quality and frequency of immunization activities to stop and prevent poliovirus transmission in the high-risk area of northwest Nigeria. PLoS One. 2015;10(6), e0130123.

5. Kalkowska DA, Duintjer Tebbens RJ, Thompson KM. Modeling strategies to increase population immunity and prevent poliovirus transmission in two high-risk areas in northern India. J Infect Dis. 2014;210(S1):398-411.
6. Kalkowska DA, Duintjer Tebbens RJ, Thompson KM. Modeling strategies to increase population immunity and prevent poliovirus transmission in the high-risk area of northwest Nigeria. J Infect Dis. 2014;210(S1):412-23.

7. Jafari H, Deshpande JM, Sutter RW, Bahl S, Verma H, Ahmad M, et al. Polio eradication. Efficacy of inactivated poliovirus vaccine in India. Science. 2014;345(6199):922-5.

8. John J, Giri S, Karthikeyan AS, Iturriza-Gomara M, Muliyil J, Abraham A, et al. Effect of a single inactivated poliovirus vaccine dose on intestinal immunity against poliovirus in children previously given oral vaccine: an open-label, randomised controlled trial. Lancet. 2014;384(9953):1505-12.

9. Duintjer Tebbens RJ, Pallansch MA, Kalkowska DA, Wassilak SG, Cochi SL, Thompson KM. Characterizing poliovirus transmission and evolution: Insights from modeling experiences with wild and vaccine-related polioviruses. Risk Anal. 2013;23(4):703-49.

10. Kalkowska DA, Duintjer Tebbens RJ, Grotto I, Shulman LM, Anis E, Wassilak SGF, et al. Modeling options to manage type 1 wild poliovirus imported into Israel in 2013. J Infect Dis. 2015;211(11):1800-12.

11. Grassly NC, Fraser C, Wenger J, Deshpande JM, Sutter RW, Heymann DL, et al. New strategies for the elimination of polio from India. Science. 2006;314(5802):1150-3.

12. Upfill-Brown AM, Lyons HM, Pate MA, Shuaib F, Baig S, Hu H, et al. Predictive spatial risk model of poliovirus to aid prioritization and hasten eradication in Nigeria. BMC Med. 2014;12:92.

13. Grassly NC, Wenger J, Durrani S, Bahl S, Deshpande JM, Sutter RW, et al. Protective efficacy of a monovalent oral type 1 poliovirus vaccine: a case-control study. Lancet. 2007;369(9570):1356-62.

14. Thompson KM, Pallansch MA, Duintjer Tebbens RJ, Wassilak SGF, Cochi SL. Modeling population immunity to support efforts to end the transmission of live polioviruses. Risk Anal. 2013;33(4):647-63.

15. Thompson KM, Kalkowska DA, Duintjer Tebbens RJ. Managing population immunity to reduce or eliminate the risks of circulation following the importation of live polioviruses. Vaccine. 2015;33(3):1568-77.

16. Thompson KM, Kalkowska DA, Duintjer Tebbens RJ. Managing population immunity to reduce or eliminate the risks of circulation following the importation of live polioviruses. Vaccine. 2015;33(3):1568-77.

17. Duintjer Tebbens RJ, Kalkowska DA, Wassilak SGF, Pallansch MA, Cochi SL, Thompson KM. The potential impact of expanding target age groups for polio immunization campaigns. BMC Infect Dis. 2014;14:45.

18. Wagner BG, Behrend MR, Klein DJ, Upfill-Brown AM, Eckhoff PA, Hu H. Quantifying the impact of expanded age group campaigns for polio eradication. PLoS One. 2014;9(12), e113538.

19. Kalkowska DA, Duintjer Tebbens RJ, Pallansch MA, Cochi SL, Wassilak SGF, Thompson KM. Modeling undetected live poliovirus circulation after apparent interruption of transmission: Implications for surveillance and vaccination. BMC Infect Dis. 2015;15(66):1.

20. Duintjer Tebbens RJ, Pallansch MA, Wassalik SGF, Cochi SL, Thompson KM: An economic analysis of poliovirus risk management policy options for 2013-2052. doi:10.1186/s12879-015-1112-8 BMC Inf Dis 2015

21. Thompson KM, Pallansch MA, Duintjer Tebbens RJ, Wassilak SG, Kim J-H, Cochi SL. Pre-eradication vaccine policy options for poliovirus infection and disease control. Risk Anal. 2013;33(4):516-43.

22. Duintjer Tebbens RJ, Pallansch MA, Kim J-H, Burns CC, Kew OM, Oberste MS, et al. Review: Oral poliovirus vaccine evolution and insights relevant to modeling the risks of circulating vaccine-derived polioviruses (cVDPVs). Risk Anal. 2013;23(4):680-702.

23. National Population Commission Nigeria and ICF International. Nigeria Demographic and Health Survey 2013. Abuja: NPC and ICF International; 2014.

24. World Health Organization: WHO/UNICEF estimated coverage time series. 2014. http://apps.who.int/immunization_monitoring/globalsummary/ timeseries/tswucoveragepol3.html. Accessed May 292014.

25. Sutter RW, John TJ, Jain H, Agarkhedkar S, Ramanan PV, Verma H, et al. Immunogenicity of bivalent types 1 and 3 oral poliovirus vaccine: a randomised, double-blind, controlled trial. Lancet. 2010;376(9753):1682-8.

26. Estívariz CF, Anand A, Gary Jr HE, Rahman M, Islam J, Bari TI, et al. Immunogenicity of three doses of bivalent, trivalent, or type 1 monovalent oral poliovirus vaccines with a 2 week interval between doses in Bangladesh: an open-label, non-inferiority, randomised, controlled trial. Lancet Infect Dis. 2015;15(8):898-904.

27. Grassly NC, Jafari H, Bahl S, Durrani S, Wenger J, Sutter RW, et al. Mucosal immunity after vaccination with monovalent and trivalent oral poliovirus vaccine in India. J Infect Dis. 2009;200(5):794-801. 
28. Duintjer Tebbens RJ, Pallansch MA, Chumakov KM, Halsey NA, Hovi T, Minor $P D$, et al. Expert review on poliovirus immunity and transmission. Risk Anal. 2013;33(4):544-605.

29. World Health Organization Collaborative Study Group on Oral Poliovirus Vaccine. Factors affecting the immunogenicity of oral poliovirus vaccine: a prospective evaluation in Brazil and the Gambia. J Infect Dis. 1995;171(5):1097-106.

30. Patriarca PA, Wright PF, John TJ. Factors affecting the immunogenicity of oral poliovirus vaccine in developing countries: review. Rev Infect Dis. 1991;13:926-39.

31. Duintjer Tebbens RJ, Pallansch MA, Chumakov KM, Halsey NA, Hovi T, Minor $\mathrm{PD}$, et al. Review and assessment of poliovirus immunity and transmission: Synthesis of knowledge gaps and identification of research needs. Risk Anal. 2013;33(4):606-46.

32. Mayer BT, Eisenberg JN, Henry CJ, Gomes MG, lonides EL, Koopman JS. Successes and shortcomings of polio eradication: a transmission modeling analysis. Am J Epidemiol. 2013:177(11):1236-45.

33. Hird TR, Grassly NC. Systematic review of mucosal immunity induced by oral and inactivated poliovirus vaccines against virus shedding following oral poliovirus challenge. PLoS Pathog. 2012;8(4), e1002599.

34. Global Polio Eradication Initiative-circulating vaccine-derived poliovirus cases 2000-2015. 2015. http://www.polioeradication.org/Dataandmonitoring/ Poliothisweek/Circulatingvaccinederivedpoliovirus.aspx. Accessed July 282015.

\section{Submit your next manuscript to BioMed Central and take full advantage of:}

- Convenient online submission

- Thorough peer review

- No space constraints or color figure charges

- Immediate publication on acceptance

- Inclusion in PubMed, CAS, Scopus and Google Scholar

- Research which is freely available for redistribution 